\title{
A new experimental technique for positive ion drift velocity measurements in noble gases: Results for xenon ions in xenon
}

\author{
P.N.B. Neves ${ }^{\mathrm{a}, *}$, C.A.N. Conde ${ }^{\mathrm{a}}$, L.M.N. Távora ${ }^{\mathrm{a}, \mathrm{b}}$ \\ ${ }^{a}$ Centro de Instrumentação, Unidade 217/94, Departamento de Física, Universidade de Coimbra, P-3004-516 Coimbra, Portugal \\ ${ }^{\mathrm{b}}$ ESTG, Instituto Politécnico de Leiria, Morro do Lena-Alto Vieiro, 2411-901 Leiria, Portugal
}

Available online 13 May 2007

\begin{abstract}
A new technique is described for measuring the drift velocities and mobilities of noble gas ions in noble gases that makes use of a gaseous electron multiplier (GEM) and a UV flash lamp to produce the ions in a thin, well-defined, planar region. The drift velocities are determined from measurements of the transit time of the ions in crossing the drift space. We present experimental results for xenon ions in xenon. For these ions, the reduced mobility values measured at various pressures when $E / N \rightarrow 0$ fall within the interval $[0.70,0.74] \mathrm{cm}^{2} \mathrm{~V}^{-1} \mathrm{~s}^{-1}$. A discussion of the results concerning the existence of $\mathrm{Xe}^{+}, \mathrm{Xe}_{2}^{+}, \mathrm{Xe}_{3}^{+}$, etc., ions is also made.

(C) 2007 Elsevier B.V. All rights reserved.
\end{abstract}

PACS: 51.20; 29.40.C; 51.10; 52.25.Fi

Keywords: Drift velocity; Xenon ions; Gas detectors; Ion mobility

\section{Introduction}

Knowledge of the drift velocities of positive ions in noble gases at atmospheric pressures and reduced electric fields of a few $T \mathrm{~d}$ (Townsend, $1 T \mathrm{~d}=10^{-21} \mathrm{~V} \mathrm{~m}^{2}$ ) or lower is of great importance in a variety of fields like gaseous radiation detectors and ion mobility spectrometry [1,2].

Indeed, in radiation detectors based on avalanche processes in gases, such as proportional counters, multiwire chambers, microstrip plate chambers, etc., the drift of positive ions near the anode plays a role in the pulse formation nearly as significant as the drift of the electrons. Therefore, to model and fully understand the physics of these types of radiation detectors, it is important to access detailed information on the transport of electrons and ions in detector gases, namely drift velocities and longitudinal and transversal diffusion coefficients.

Consider a collection of ions in a gas of uniform temperature and constant pressure. If a uniform electric field is applied throughout the gas, the velocity of the centre of charge of the ion cloud is called the drift velocity,

\footnotetext{
*Corresponding author.

E-mail address: pneves@saturno.fis.uc.pt (P.N.B. Neves).
}

$v_{\mathrm{d}}$, and it is directly proportional to the electric field intensity $E[1]$. Thus

$v_{\mathrm{d}}=K E$

where the constant of proportionality $K$ is the mobility of the ions. To facilitate the comparison and use of data, the mobility $K$ is usually converted into a "reduced mobility" $K_{0}$, defined by the equation

$K_{0}=\frac{K N}{N_{0}}$

where $N$ is the gas number density and $N_{0}$ its value at $273 \mathrm{~K}$ and $1 \mathrm{~atm}\left(N_{0}=2.687 \times 10^{19} \mathrm{~cm}^{-3}\right)$.

While searching for data concerning the drift of positive ions in detector gases, namely noble gases at atmospheric pressures and low reduced electric fields, we realized that the data available in the scientific literature is scarce. So we embarked on a research programme to obtain ion transport data, both theoretical and experimental, for noble gas ions in noble gases [3-5]. The calculations we carried out up to the present moment deal only with the transport of single-charge, atomic noble gas ions in noble gases, like $\mathrm{Xe}^{+}$in xenon [5]. However, there is evidence $[6,7]$ that, at atmospheric pressures, molecular noble gas 
ions like $\mathrm{Xe}_{2}^{+}, \mathrm{Xe}_{3}^{+}$, etc., are formed in processes like

$\mathrm{Xe}^{+}+\mathrm{Xe}+\mathrm{Xe} \rightarrow \mathrm{Xe}_{2}^{+}+\mathrm{Xe}$

$\mathrm{Xe}_{2}^{+}+\mathrm{Xe}+\mathrm{Xe} \rightarrow \mathrm{Xe}_{3}^{+}+\mathrm{Xe}$

The scope of this work is to measure the effective average drift velocities of $\mathrm{Xe}^{+}, \mathrm{Xe}_{2}^{+}, \mathrm{Xe}_{3}^{+}$, etc., ions in xenon. We report the results obtained for different pressures and compare them with the few existing data, both theoretical and experimental.

\section{Experimental set-up}

The technique that we have employed uses a gaseous electron multiplier (GEM) [8] to produce the ions. The GEM has a thin gold layer that acts as a photocathode. A xenon VUV flash lamp releases photoelectrons from the gold layer, which trigger an electron avalanche in the GEM (Fig. 1). The ions that are produced during the avalanche will then drift across a uniform electric field region towards a collection grid, where they induce, while drifting, a linearly growing charge pulse.

A schematic view of the set-up used in the present work is shown in Fig. 2. The chamber body is made of stainless steel. Macor pieces fixed with low vapour pressure epoxy to the detector body were used for insulating the feedthroughs of the detector biasing. For simplicity, only two of the three feedthroughs assembled are depicted in Fig. 2. The other feedthrough is connected to the remaining electrode of the GEM. The top and the bottom electrodes of the GEM are connected to a dual high-voltage supply (CAEN-2 Fold HV Power Supply Mod. N471). The chamber body was grounded.

The vessel was connected to a vacuum/gas-filling system and sealed during the experiments. It was filled with xenon, continuously purified through getters (SAES St-707/ washer/833) at $150{ }^{\circ} \mathrm{C}$ and maintained in circulation by convection. The getters will absorb chemical active impurities such as $\mathrm{H}_{2}, \mathrm{O}_{2}, \mathrm{H}_{2} \mathrm{O}, \mathrm{CO}, \mathrm{CO}_{2}$ and $\mathrm{N}_{2}$. The gas pressure was measured using a MKS gauge (model 722A53THA3FC) with an accuracy of $1 \%$.

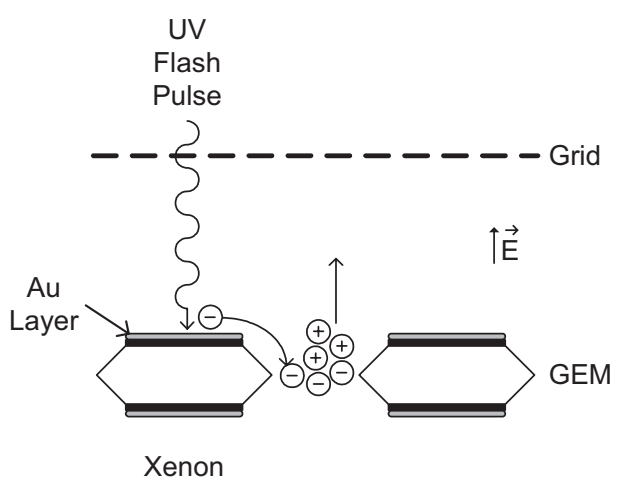

Fig. 1. Schematic diagram of the physical processes that lead to the production and detection of the ions.

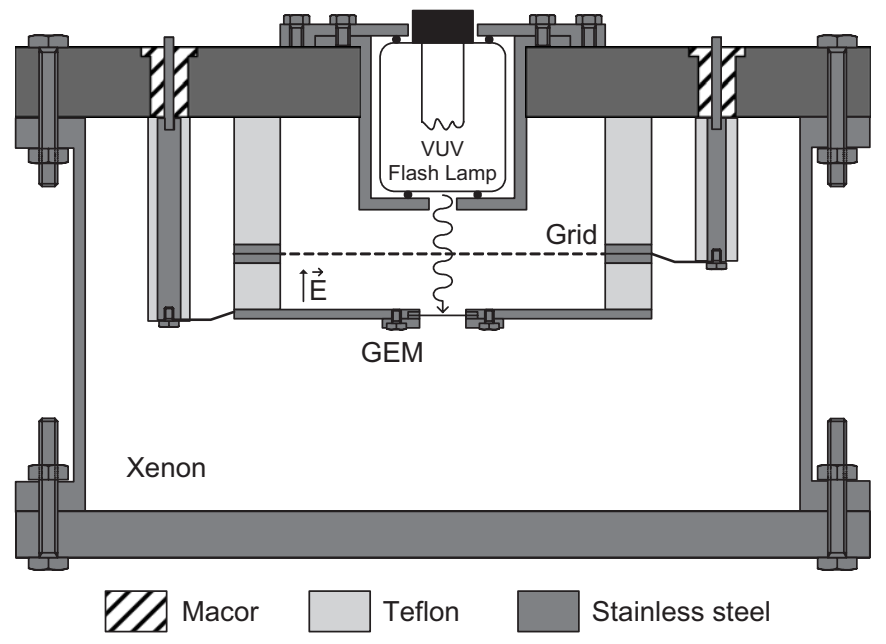

Fig. 2. Schematic view of the experimental set-up.

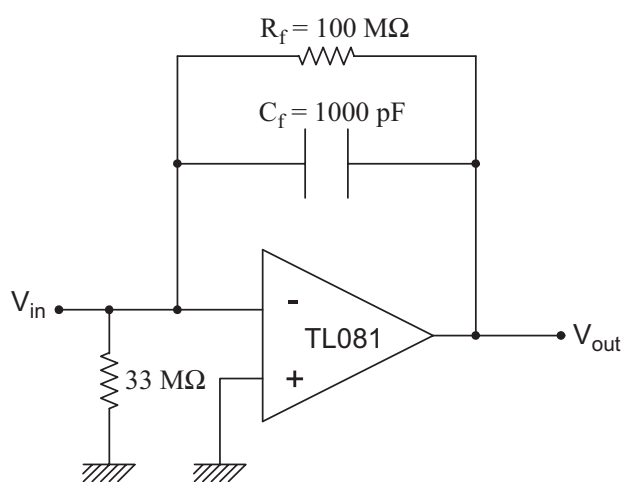

Fig. 3. Charge preamplifier for the ion pulses.

The grid and the GEM are separated by $11.2 \pm 0.1 \mathrm{~mm}$ using Teflon spacers. The GEM size is $10 \times 10 \mathrm{~mm}^{2}$, one of the standard types produced at CERN; the holes have a double conical shape with an inner diameter of 50 and $80 \mu \mathrm{m}$ at the metal surface. The holes are arranged in an hexagonal pattern with a pitch of $140 \mu \mathrm{m}$. The thickness of the GEM is about $60 \mu \mathrm{m}: 50 \mu \mathrm{m}$ thick Kapton with $5 \mu \mathrm{m}$ copper on each side. The grid wires are $80 \mu \mathrm{m}$ in diameter, spaced by $1 \mathrm{~mm}$.

A Hamamatsu xenon VUV flash lamp (model L2439) was used and operated at a repetition rate of $10 \mathrm{~Hz}$. The flash pulse width (FWHM) is inferior to $15 \mu \mathrm{s}$ and the maximum average power energy per flash is $0.1 \mathrm{~J} /$ flash.

The signal is amplified using the charge pre-amplifier shown in Fig. 3 and recorded in a digital oscilloscope (Tektronix TDS 1012). The feedback resistor $\left(R_{\mathrm{f}}\right)$ and capacitor $\left(C_{\mathrm{f}}\right)$ were chosen so that the decay time ( $\left.\tau=R_{\mathrm{f}} C_{\mathrm{f}}=0.1 \mathrm{~s}\right)$ is much longer than the duration of the longest ion impulse recorded. Measuring the time that the ions take to travel the distance between the GEM and the grid allows for the calculation of their drift velocities (see Fig. 4). 


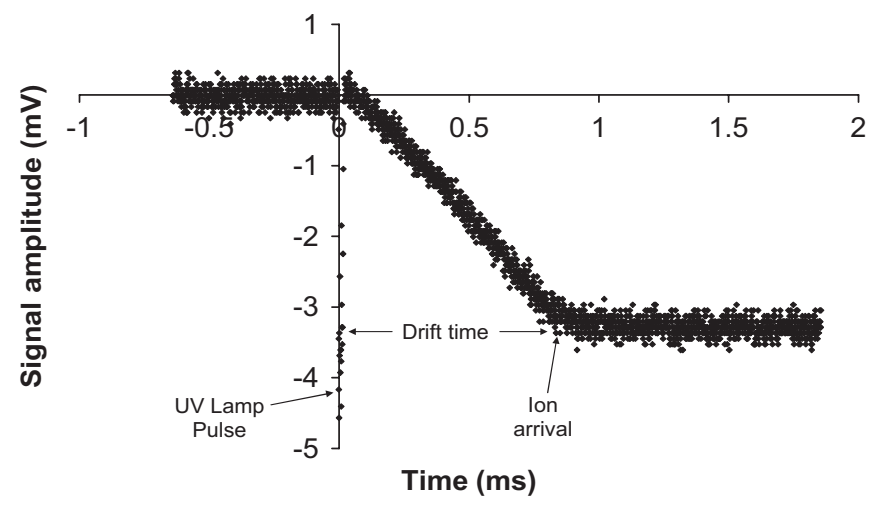

Fig. 4. Typical average of 128 digitalized ion induced pulses.

\section{Results and discussion}

We have measured the drift velocity of xenon ions in xenon for different reduced electric fields $\mathrm{E} / \mathrm{N}$ (below $10 T d)$ and for different pressures (1, 0.5, 0.25 and $0.125 \mathrm{~atm})$. The results are represented in Fig. 5, where the errors were estimated to be less than $5 \%$. We then calculated by extrapolation the reduced mobilities when $E / N \rightarrow 0$ and the values fall within the interval $[0.70,0.74] \mathrm{cm}^{2} \mathrm{~V}^{-1} \mathrm{~s}^{-1}$.

Above 5 Torr virtually all $\mathrm{Xe}^{+}$are converted to the molecular species $\mathrm{Xe}_{2}^{+}$soon after being created [7]. According to the same article where the equilibrium constant of reaction (4) is reported, at atmospheric pressures $\mathrm{Xe}_{3}^{+}$ions are very abundant. Taking into account the equilibrium constant indicated in Ref. [7], at 1 atm the proportion of $\mathrm{Xe}_{2}^{+}$and $\mathrm{Xe}_{3}^{+}$ions is $1: 10\left(1 \mathrm{Xe}_{2}^{+}\right.$to $\left.10 \mathrm{Xe}_{3}^{+}\right)$ and at $0.125 \mathrm{~atm}$ it is $8: 10$. The number of $\mathrm{Xe}^{3+}$ ions grows with the increasing pressure. However, there is no information on the time reaction (4) takes to reach equilibrium. If it is much longer than the typical ion drift time, few $\mathrm{Xe}^{3+}$ ions will be formed before the ion cloud reaches the cathode.

There are very little data available on the mobility of $\mathrm{Xe}_{3}^{+}$ions. The only data that we found, report on the mobility of $\mathrm{Xe}_{3}^{+}$ions at a temperature of $273 \mathrm{~K}$ [7] and we conducted our experiments at $300 \mathrm{~K}$. At $273 \mathrm{~K}, \mathrm{Xe}_{3}^{+}$ions are about $15 \%$ slower than the $\mathrm{Xe}_{2}^{+}$ions. However, while the mobility of the $\mathrm{Xe}_{2}^{+}$ions diminishes with the temperature, the mobility of the $\mathrm{Xe}_{3}^{+}$ions increases with the temperature. So, at $300 \mathrm{~K}$ we should expect a smaller difference between the mobilities of the two ion species.

Previous attempts to measure the mobility of $\mathrm{Xe}_{2}^{+}$ ions were made by Varney [9], Biondi and Chanin [10] and Helm [7], and the calculated reduced mobilities when $E / N \rightarrow 0$ are indicated in Table 1 .

Our results are in good agreement with the data from Varney indicating that we might be in the presence of $\mathrm{Xe}_{2}^{+}$ ions.

Although the quantity of $\mathrm{Xe}_{2}^{+}$and $\mathrm{Xe}_{3}^{+}$ions is pressuredependent, the results from Fig. 5 reveal that there is no

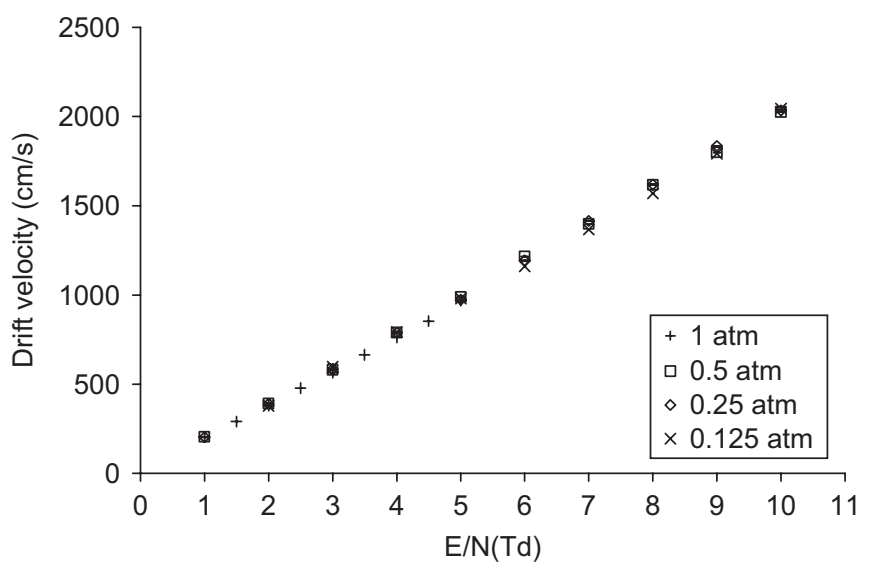

Fig. 5. Drift velocity of the xenon ions as a function of the reduced electric field.

Table 1

Previous attempts to measure the mobility of $\mathrm{Xe}_{2}^{+}$ions.

\begin{tabular}{llll}
\hline Authors & $\begin{array}{l}\mathrm{E} / \mathrm{N} \text { range } \\
(T \mathrm{~d})\end{array}$ & $\begin{array}{l}\text { Temperature } \\
(K)\end{array}$ & $\begin{array}{l}K_{0}(E / N \rightarrow 0) \\
\left(\mathrm{cm}^{2} \mathrm{~V}^{-1} \mathrm{~s}^{-1}\right)\end{array}$ \\
\hline $\begin{array}{l}\text { Varney } \\
\text { Biondi/Chanin }\end{array}$ & {$[130,210]$} & 300 & {$[0.67,0.77]$} \\
Helm & {$[1,240]$} & $\begin{array}{l}\text { Not known } \\
293]\end{array}$ & 0.79 \\
\hline
\end{tabular}

significant variation in the ions drift velocity with the pressure of the gas, for the same $E / N$. This can mean either that reaction (4) is very slow and so there are few $\mathrm{Xe}_{3}^{+}$ions or that the reduced mobilities of the two ions species are very similar, making them indistinguishable for this experimental technique.

\section{Conclusions}

We have developed a new technique for the measurement of ion mobilities in gases, which for xenon ions in xenon gives results similar to those presented by Varney [9] for the $\mathrm{Xe}_{2}^{+}$ions. However, according to Ref [7] and for the pressures considered in this work, $\mathrm{Xe}_{3}^{+}$ions are more abundant than the $\mathrm{Xe}_{2}^{+}$ions.

There is almost no information on $\mathrm{Xe}_{3}^{+}$ions in the scientific literature, but because no significant variation in the ion drift velocity with the gas pressure was observed, one can infer that either the velocities of $\mathrm{Xe}_{2}^{+}$and $\mathrm{Xe}_{3}^{+}$are very similar, or reaction (4) is very slow and few $\mathrm{Xe}_{3}^{+}$ions are formed during the ion cloud drift time.

\section{Acknowledgments}

This work was supported by FEDER and Fundação para a Ciência e Tecnologia (FCT) through project POCI/ FP/63430/2005. P.N.B. Neves was supported by an FCT grant (SFRH/BD/18926/2004). Thanks are due to Prof. Filomena P. Santos for helpful discussions. 


\section{References}

[1] G.F. Knoll, Radiation Detection and Measurement, second ed., Wiley, 1989.

[2] G.A. Eiceman, E.G. Nazarov, J.E. Rodriguez, Rev. Sci. Instr. 72 (2001) 3610

[3] J.A.S. Barata, C.A.N. Conde, Radiat. Phys. Chem. 71 (2004) 647.
[4] J.A.S. Barata, C.A.N. Conde, Radiat. Phys. Chem. 71 (2004) 721.

[5] J.A.S. Barata, C.A.N. Conde, IEEE Trans. Nucl. Sci. 52 (2005) 2889.

[6] A.P. Vitols, H.J. Oakam, Phys. Rev. A 8 (1973) 1860.

[7] H. Helm, Phys. Rev. A. 14 (1976) 680.

[8] F. Sauli, Nucl. Instr. and Meth. A 386 (1997) 531.

[9] R.N. Varney, Phys. Rev. 88 (1952) 362.

[10] M.A. Biondi, L.M. Chanin, Phys. Rev. 94 (1954) 910. 